Scientific Paper

\title{
Toward a better prescription method for external radiotherapy
}

\author{
Marzena MROZOWSKA ${ }^{1, a}$, Paweł KUKOŁOWICZ ${ }^{2}$ \\ ${ }^{1}$ Szpital Wojewodzki im. Sw. Lukasza, Tarnow, Poland \\ ${ }^{2}$ Medical Physics Department, Cancer Center-Institute of Oncology, Warsaw, Poland \\ ${ }^{a}$ E-mail address: marzena.mrozowska5@gmail.com
}

(received 13 February 2017; accepted 3 March 2017)

\begin{abstract}
Aim: The aim of the study was to compare several methods of dose prescription, the mean dose, the median dose, the effective dose and the generalized Equivalent Uniform Dose (gEUD).

Background: The dose distribution in the planning target volume is never fully homogenous. Depending on the dose prescription method for the same prescribed dose different biologically equivalent doses are delivered. The latest ICRU Report 83 proposes to prescribe the dose to the median dose in the PTV. Several other methods are also in common use. It is important to know what are differences of doses actually delivered depending on the dose prescription method.

Materials and methods: The study was performed for three groups of patients treated radically with external beams in Brzozow, over the 2012-2013 period. The groups were of patients with breast, lung and prostate cancer. There were 10 patients in each group. For each patient all metrics, i.e. the mean dose, the median dose, the effective dose and the generalized Equivalent Uniform Dose, were calculated. The influence of the dose homogeneity in the PTV on the results is also evaluated. The gEUD was used as a reference dose prescription method.

Results: For all patients, an almost perfect correlation between the median dose and the gEUD was obtained. Worse correlation was obtained between other metrics and the gEUD. The median dose is almost always a little higher than the gEUD, but the ratio of these two values never exceeded 1.013.

Conclusion: The median dose seems to be a good and simple method of dose prescription.
\end{abstract}

Key words: radiotherapy; dose; equivalent uniform dose.

\section{Introduction}

The goal of the treatment planning is to conform the dose distribution to the target volume and to reduce the dose to sensitive structures and healthy tissues as much as possible. Yet it is widely accepted that the resultant dose distribution in each of the planning target volumes should be as homogenous as possible. Uniformity of dose distribution depends on many factors: on the geometric relation between critical structures and the target, the planning method, the skills of the planners and others. Very seldom can the dose distribution in the target volume may be treated as uniform. Lack of full uniformity leads to the problem of dose prescription. For several years, the delivered dose was prescribed to the minimum dose, or the so called envelope isodose [1]. For obvious reasons, this prescription leads to underestimation of the actual dose - the delivered dose was larger, in many cases much larger than the prescribed one. The ICRU Report 50 proposed to prescribe the dose to the ICRU reference dose, which is the dose delivered to the point located in the center of the planning target volume [2]. At approximately the same time, the Nordic Association of Clinical Physicists proposed to prescribe the dose to the mean dose to the target volume [3]. This idea was based on the work published by Brahme. Anders Brahme showed that if the dose distribution in the target volume is uniform enough, i.e. the standard deviation of dose distribution is smaller than approximately $3 \%$ of the mean dose, then the effective dose delivered to the target can be approximated by the mean target dose. The term "effective dose" was used in the sense proposed by Andrzej Niemierko [4]. Niemierko introduced the idea of the so called Equivalent Uniform Dose (EUD) which is defined as the biologically equivalent dose which, if given uniformly, leads to the same cell kill in the tumor volume as an actual (non-uniform) dose distribution. Brahme described the effective dose with the formulae [5]:

$D_{\text {eff }}=D_{\text {mean }} \cdot\left[1-\frac{\gamma}{2 \cdot T C P\left(D_{\text {mean }}\right)} \cdot\left(\frac{\sigma}{D_{\text {mean }}}\right)^{2}\right]$

Eq. 1

Where:

$\mathrm{D}_{\text {mean }}$ - the mean dose;

$\gamma$ - normalized dose gradient;

$\mathrm{TCP}\left(\mathrm{D}_{\text {mean }}\right)$ - tumor control probability for dose $\mathrm{D}_{\text {mean }}$;

$\sigma-$ standard deviation of dose distribution in the PTV.

This expression shows that the effective dose decreases below $D_{\text {mean }}$ if the relative standard deviation of the dose 
distribution increases. For normalized dose gradients observed clinically, for standard deviations smaller than $3 \%$, there is negligible influence of the non-uniformity on the treatment outcome. Niemierko's idea, the Equivalent Uniform Dose [4], was eventually described in a very simple mathematical form and called as the general Equivalent Uniform Dose [6]. The advantage of the generalized form over the Equivalent Uniform Dose is that the same formulae may be used also for normal tissues [6,7]:

$$
g E U D=\left(\frac{1}{K} \sum_{k} D_{k}^{a}\right)^{\frac{1}{a}}
$$

Where:

$D_{k}$ are the doses delivered to each single voxel of the PTV or an Organ at Risk;

a - parameter which describe the dose-volume effect.

In daily practice the mathematically oriented methods of dose distribution evaluation are used very seldom. In the vast majority of hospitals the dose distribution in the PTV is analyzed graphically in the form of the Dose Volume Histogram (DVH) and with the simple dose distribution metrix. The generalized EUD is rather seldom applied for 3D conformal radiotherapy, more often for IMRT [8,9]. According to the ICRU Report 83, the dose should be prescribed to the median dose [10].

In this study, the gEUD has been used as the gold standard and other metrics, i.e. the mean dose, the median dose in the PTV and the effective dose to the PTV were compared with the gEUD.

\section{Materials and methods}

The study was performed for three groups of patients treated radically with external beams in Brzozow over the 2012-2013 period. There were patients with breast, lung and prostate cancer. There were 10 patients in each group, altogether 30 patients. More details on the clinical material may be found elsewhere [11]. The plans were CT based plans. The CT examinations were performed in treatment position (Somatom Open, Siemens) with 3-5 mm slice thickness in spiral mode. For each patient, the Clinical Target Volume and organs at risk were delineated. The treatment planning was performed with the Xio (Elekta, version 4.70.00) treatment planning system. The contours of the PTV and organs at risk were prepared according to the ICRU 50. The dose was always prescribed to the ICRU Reference Point. The superposition algorithm with a calculation grid of $0.25 \mathrm{~cm}$ was used.

For each patient the following metrics in the PTV were obtained:

- maximum dose $\left(D_{\max }\right)$; the maximum dose in a single calculation point,

- minimum dose $\left(\mathrm{D}_{\min }\right)$; according to ICRU Report 50 [2],

- the mean dose $\left(\mathrm{D}_{\text {mean }}\right)$,
- the effective dose $\left(D_{\text {eff }}\right)$; see the Equation 1 in the Introduction. To calculate the $\mathrm{D}_{\text {eff }}$ we assumed that the TCPs for the breast, lung and prostate were $0.8,0.2$, and 0.8 , respectively. To calculate $D_{\text {eff }}$, it was assumed that $\gamma=1.0$. Calculation of the effective dose was additionally done for gamma $=1.5$ and 2 ,

- median dose $\left(\mathrm{D}_{50 \%}\right)$; according to ICRU Report 83 [10],

- general Equivalent Uniform Dose with $\mathrm{a}=-7,2$ for breast cancer, $\mathrm{a}=-13$ for lung cancer, $\mathrm{a}=-10$ for prostate cancer [7]; see Equation 1 in the Introduction.

The correlations were calculated for:

- the median dose and the gEUD,

- the mean dose and gEUD,

- the effective dose and gEUD.

The homogeneity of dose distribution in the target volume was evaluated in terms of the difference between the maximum and minimum dose (according to the ICRU 50 Report).

\section{Results}

For presentation purposes, the values were always given as the ratio of a given result and the prescribed dose. In Figure 1, the correlation between the median dose and the gEUD is shown. There is an almost perfect correlation between these two terms. The $\mathrm{D}_{50 \%}$ was higher than the gEUD for 28 of 30 patients, however, the ratio of these two values never exceeded 1.013. The mean value of the ratios was $1.004 \pm 0.003$.

Much worse correlations were obtained for the other two pairs of dose distribution indices. In Figure 2, the correlation between the mean dose delivered to the PTV and the gEUD is shown. There was poor correlation between the mean dose delivered to the PTV and the gEUD. For some patients the mean dose is higher and for other lower than the gEUD.

In Figure 3, the correlation between the effective dose Deff to the PTV and the gEUD is shown. The correlation between the effective dose delivered to the PTV and the gEUD was also poor. Calculation of the effective dose was also carried out for gamma $=1.5$ and 2. Very similar results were obtained.

The separate analysis of the data for each location also showed a very good correlation between the gEUD and the median dose. In Figures 4, 5, and 6, we present these correlations.

\section{Discussion}

Dose distribution in the Planning Target Volume is never fully homogenous. For some patients, the dose distribution is quite homogenous, but for others may be quite inhomogeneous. The individual anatomy and the position of the target with respect to organs at risk influences the homogeneity of dose distribution most of all. It is much more difficult to obtain the homogenous dose distribution for lung patients than for prostate patients. Therefore to prescribe and report the dose delivered to the target we must choose a statistic of dose distribution in the PTV. 


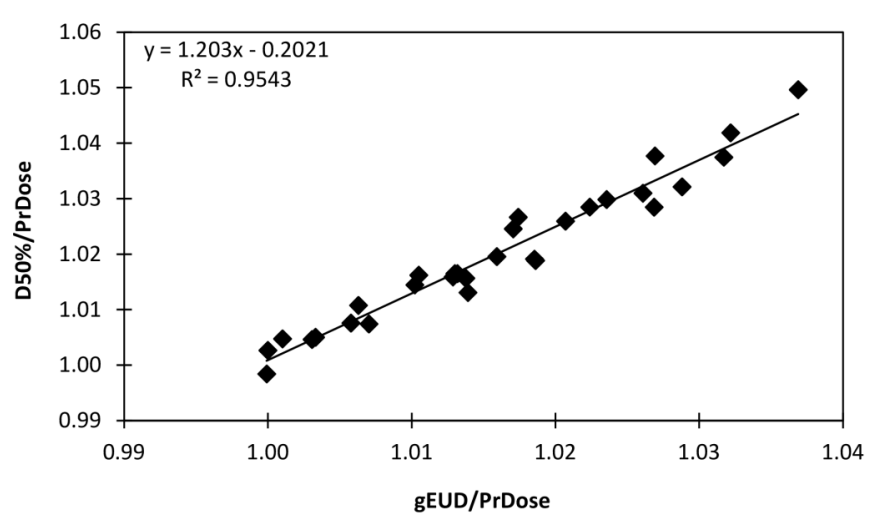

Figure 1. Correlation between the gEUD and $D_{50 \%}$ for 30 patients.

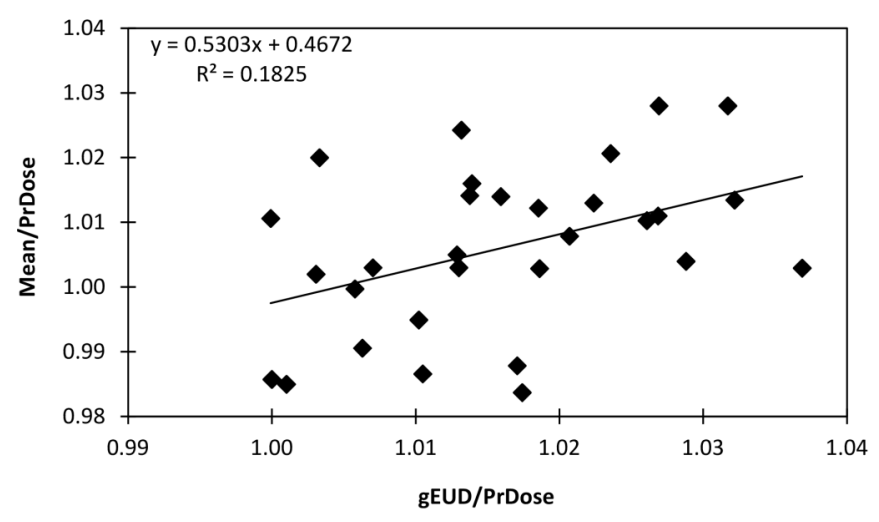

Figure 2. Correlation between the gEUD and the mean dose delivered to the PTV.

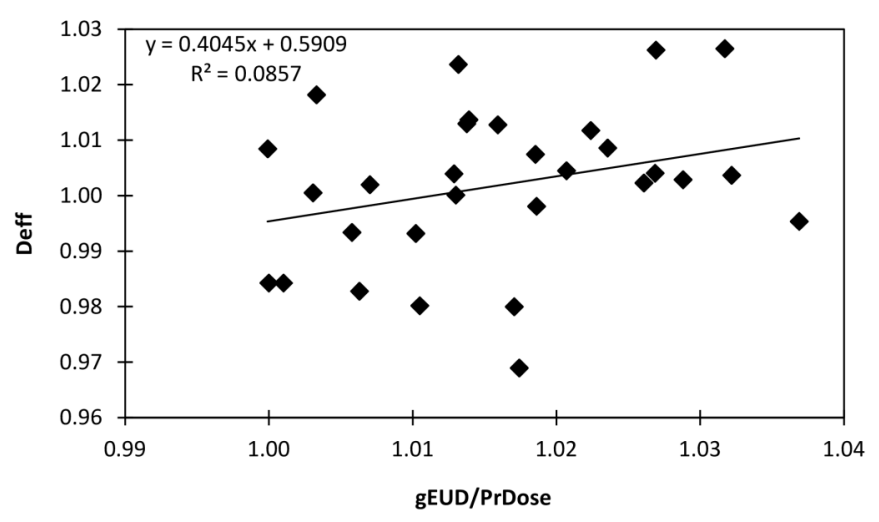

Figure 3. Correlation between the gEUD and the effective dose delivered to the PTV.

According to ICRU 50, the dose should be prescribed and reported to the ICRU Reference Point. The NACP proposed to prescribe and report the delivered dose to the mean dose to the PTV. The ICRU Report 83 recommend to prescribe the dose to the median dose to the PTV. Theoretically, it would be best to use the dose statistic which is well correlated with the Tumour Control Probability. This was the reason of using the Niemierko's idea. Niemierko proposed the model in which the non-uniform dose distribution is recalculated to the homogenous one, for which the Tumour Control Probability is the same as for the actual one. His concept of the Equivalent

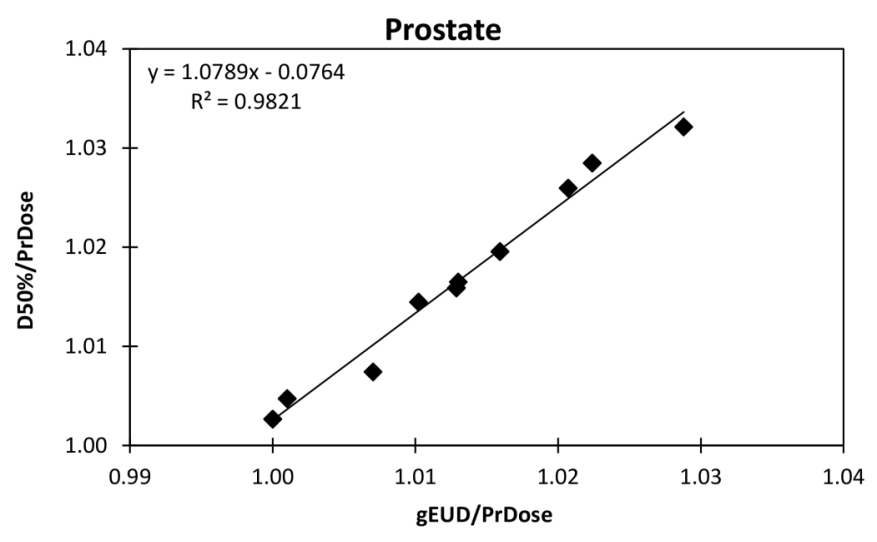

Figure 4. Correlation between the gEUD and $D_{50 \%}$ for prostate patients.

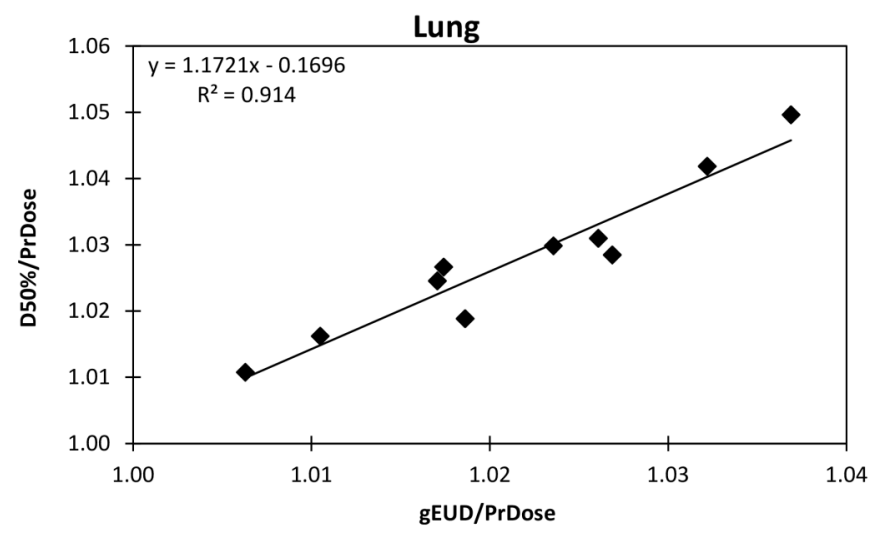

Figure 5. Correlation between the gEUD and $D_{50 \%}$ for lung patients.

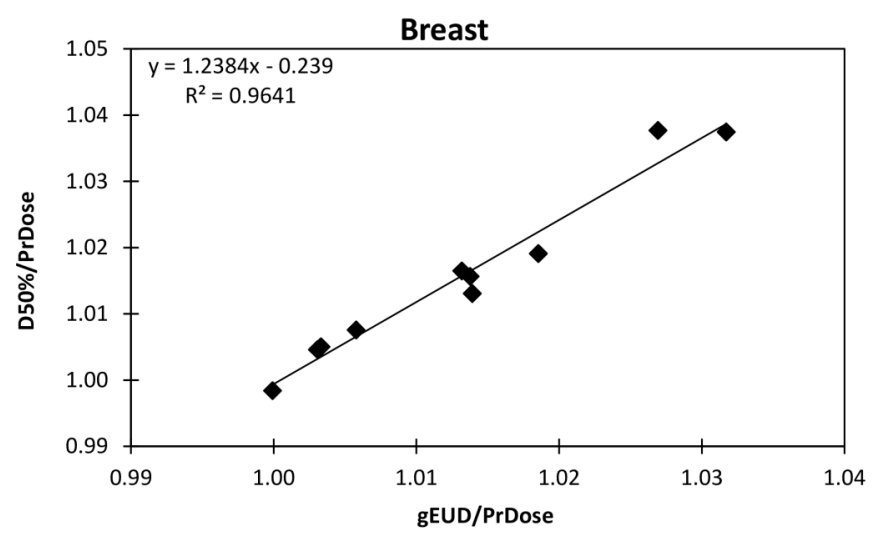

Figure 6. Correlation between the gEUD and $D_{50 \%}$ for breast patients.

Uniform Dose is used rather seldom in the clinic for several reasons. The main reason is because to calculate the EUD quite complex calculations must be performed. However, one may observe that mathematical models are more and more often used, at least in research $[12,13,14]$. In this work, we compared the ICRU Reference Dose, the effective dose, the mean dose and the median dose with the Equivalent Uniform Dose for three group of patients. These were patients irradiated for prostate, lung and breast targets. In the first group of patients dose distributions in the PTV were quite homogenous, the mean value of differences between the maximum and 
minimum doses in the PTV and the standard deviation of dose distribution in the PTV were 9,3\% and $2,8 \%$, respectively. In the two other locations, the dose distributions were less homogenous. For lung patients, the mean value of differences between the maximum and minimum doses and standard deviation in the PTV were 17,8\%, 5,2\%; and for breast patients, they were $15,9 \%$ and $4,7 \%$ respectively. However, for all patients we obtained an almost perfect correlation between the median dose and the gEUD, regardless of which group of patients was being considered. The median dose is almost always a little larger than the gEUD but the ratio of these two values never exceeded 1.013. For two patients for whom the median dose was smaller than the gEUD, the ratios were very close to $1.0(0.999)$. We may conclude that the median dose is a very good estimate of the Equivalent Uniform Dose.

To our surprise, we did not obtain such a good correlation between the Equivalent Uniform Dose and the two other dose distribution indices, the effective dose and the mean dose. For some patients, the gEUD is larger than the mean dose and the effective dose, for some others, it is smaller. The ratio exceeded 1.03. Using these different methods of dose prescription may lead to delivering the total doses differing by one conventional fraction dose (the $2 \mathrm{~Gy}$ fraction dose is the $3 \%$ of the typical total dose of $66 \mathrm{~Gy}$ ) and consequently if we assume that the normalized dose gradient is 2 , it might lead to a TCP difference of 0.06 (0.03 x 2). For daily clinical work, this difference is not so significant but, it may influence considerably the reliability of clinical trials, where the quality of data plays an important role. In clinical trials, a simple and unambiguous, dose prescription is of special concern. Also, whenever different plans are compared, the first question concerns the dose prescription. If the doses were prescribed to different dose statistics, the subject of dose distribution comparison, which is difficult in general, would become even more complicated [15]. Prescribing the dose distribution to the median dose in the era of $3 \mathrm{D}$ treatment planning systems is a simple task.

The EUD and the median dose which is very well correlated with the EUD seems currently to be a very attractive and the most reliable method of dose prescription. However, there are at least two week points of the EUD. Proposing the EUD concept Niemierko assumed that: the dose distribution of the clonogens in the target is uniform which is not true, and that intrinsic sensitivity of all tumour cells is the same. Thanks to new methods of imaging, the Positron Emission Tomography, dynamic contrast-enhanced magnetic resonance and spectroscopy, we are able to map in three dimensions the distribution of the density of the target cells as well as to have information about the cells' sensitivity to radiation $[16,17]$. Nevertheless, in the authors opinion, using the Equivalent Uniform Dose or it surrogate, i.e. the median, dose allows to make the next step towards improved precision of dose prescription.

\section{Conclusions}

There is a very good correlation between the Equivalent Uniform Dose and the median dose to the PTV. The dose prescription to the median dose proposed by the ICRU 83 seems to be the most simple and reliable method of dose prescription.

\section{References}

[1] ICRU. Basic Aspects of High Energy Particle Interactions and Radiation Dosimetry. Bethesda, MD: International Commission on Radiation Units and Measurements, ICRU Report 28; 1978.

[2] ICRU. Prescribing, recording and reporting photon beam therapy. Bethesda, MD: International Commission on Radiation Units and Measurements, ICRU Report 50; 1993.

[3] Aaltonen P, Brahme A, Lax I, et al. Specification of dose delivery in radiation therapy. Recommendation by the Nordic Association of Clinical Physics (NACP). Acta Oncol. 1997;36(Suppl10):1-32.

[4] Niemierko A. Reporting and analyzing dose distributions: A concept of equivalent uniform dose. Med Phys. 1997; 24(1):103-110.

[5] Brahme A. Which parameters of the dose distribution are best related to the radiation response of tumours and normal tissues. In: Radiation Dose in Radiotherapy from Prescription to Delivery (Proceedings of an Interregional Seminar for Europe, the Middle East and Africa, Leuven, 16-20 September 1991). IAEA TECDOC No. 734. IAEA; 1994. p. 37-58.

[6] Niemierko A. A generalized concept of equivalent uniform dose [abstract]. Med Phys. 1999;26:1100.

[7] Gay H, Niemierko A. A free program for calculating EUD-based NTCP and TCP in external beam radiotherapy. Phys Med. 2007; 23(3-4):115-125.

[8] Mavroidis P, Lind BK, Brahme A. Biologically effective uniform dose (D) for specification, report and comparison of dose response relations and treatment plans. Phys Med Biol. 2001;46(1):2607-2630.

[9] Wu Q, Mohan R, Niemierko A, Schmidt-Ullrich R. Optimization of intensity-modulated radiotherapy plans based on the equivalent uniform dose. Int J Radiat Oncol Biol Phys. 2002;52(1):224-235.

[10] ICRU. Prescribing, recording, and reporting photon-beam intensity-modulated radiation therapy (IMRT). ICRU Report 83. J ICRU. 2010;10(1):1-106. 
[11] Mrozowska M, Kukołowicz P. Relationships between various indices of doses distribution homogeneity. Rep Pract Oncol Radiother. 2015;20(4):278-283.

[12] Miles EF, Nelson JW, Alkaissi AK, et al. Biologically effective dose (BED) correlation after low-dose rate prostate brachytherapy for clinically low-risk prostate cancer. Int J Radiat Oncol Biol Phys. 2010;77(1):139-146.

[13] Hartley A, Sanghera P, Kazi W, et al. Correlation of currently used radiobiological parameters with local control and acute and late mucosal toxicity in randomised studies of altered fractionation for locally advanced head and neck cancer. Clin Oncol. 2011;23(1):29-33.

[14] Xia B, Chen GY, Cai XW, et al. The effect of bioequivalent radiation dose on survival of patients with limited-stage small-cell lung cancer. Radiat Oncol. 2011;6:50.

[15] Piotrowski T, Jodda A. How to compare treatment plans? Personalized perspective. Rep Pract Oncol Radiother. $2015 ; 20(2): 77-78$.

[16] Bentzen SM. Theragnostic imaging for radiation oncology: dose-painting by numbers. Lancet Oncol. 2005;6(2):112-117.

[17] Toma-Dasu I, Dasu A. Towards Multidimensional Radiotherapy: key challenges for treatment individualisation. Comput Math Methods Med. 2015;2015:934380. 